\title{
Hybrid RANS-LES modeling of a strongly detached turbulent flow around a tandem cylinders configuration
}

\author{
M. Gual Skopek ${ }^{1,2}$, M. Braza ${ }^{1}$, Y. Hoarau ${ }^{3}$, F. Thiele ${ }^{2}$ \\ ${ }^{1}$ IMFT-UMR CNRS-INPT-UPS-N5502, Allée du Prof. Camille Soula, 31400 Toulouse \\ ${ }^{2}$ ISTA Dept., Technical University Berlin \\ ${ }^{3}$ IMFSS Institut de Mécanique des Fluides et de Solides de Strasbourg
}

\begin{abstract}
The turbulent flow around a generic configuration of a landing gear ('the tandem cylinder') is simulated and analysed physically at $\mathrm{Re}=1.66 \times 10^{5}$, by means of hybrid RANS-LES turbulence modelling approaches. In the present study, the Delayed Detached Eddy Simulation (DDES) approach has been employed. The DDES-OES modelling has been considered, especially involving turbulence length scale reconsiderations in the statistical part, by means of the Organised Eddy Simulation, (OES), to take into account non-equilibrium turbulence effects. The DDES- $k-\omega$ SST model is also considered. The results, obtained by means of two different time steps are compared with experiments carried out at the NASALangley Research Centre in the context of ATAAC EU-program in which the tandem cylinders is one of the 'stepping stones'. In the present study, the benefits of these hybrid approaches have been discussed for capturing the vortex dynamics and frequency modes responsible for aerodynamic noise production in the context of landing gear configurations.
\end{abstract}

\section{Introduction}

Within the ATAAC (Advanced Turbulence Simulations for Aerodynamic Application Challenges) EU program, it has been shown through its main testcases, that DDES (Delayed Detached Eddy Simulation) is a promising approach to capture the unsteady dynamics and the turbulence statistical content in strongly detached unsteady flows, by using reasonably fine grids, comparing to the grid sizes that would be needed by LES, for the present high-Reynolds number range flows around bodies, involving strong detachment. The present configuration highly interests aeroacoustics and aerodynamic noise control, generated by the two supports (tandem cylinders) of a generic configuration of a landing gear. The present test case has been the object of detailed experimental and numerical studies leaded by the NASA Langley Research Center, (Jenkins, Khorrami et al, 2005, Lockard, 2006, 2007, among other). The experiments were carried out by standard 2D PIV, at Reynolds number 166,000. In order to avoid natural transition 
Fourth Symposium on Hybrid RANS-LES Methods, 28-30 September 2011, Beijing, China

development, the experiments applied two transition strips at two specific upstream locations. A first synthesis of numerical simulations carried out for this test case was carried out by Lockhard, regrouping an order of 13 contributions involving different modelling approaches, as well as previous simulations by Khorrami et al, 2006, using URANS-SST. These simulations indicated that the majority of the approaches captured quite well the Strouhal number of the vortex shedding frequency around the first cylinder, $(\mathrm{St}=0.24)$. Furthermore, as is seen in the ATAAC European program, the DES approaches better capture the complex vortex dynamics of the present flow, especially the formation of Kelvin-Helmholz vortices in the separated shear layers. In the experimental context, it was found that the shear layers formed downstream of the first cylinder wrap around the second cylinder and interact non-linearly with the complex turbulence background, producing predominant frequencies in the energy spectrum, in the range of acoustic noise. Moreover, the numerical studies reported by Lockard in the context of the workshop for airframe noise computation, evaluated the mean drag coefficient provided by the different simulations, that had shown quite a dispersion among the different studies, with a most probable mean value of order 0.484 around the first cylinder.

The aim of the present paper is to evaluate the ability of the DDES methodology for an accurate prediction of the pressure fluctuations and frequencies responsible for the acoustic noise.

In the present study, the DDES approach is considered, involving OES modelling (Braza et al, 2006), in the URANS part, accounting for improvement of the nearregion flow physics in respect of non-equilibrium turbulence. In Bourguet et al (2008), the DES-OES modelling was successfully applied for the simulation of the strongly detached flow around an airfoil beyond stall. The present DDES-OES, as the previous one, aims at providing a smooth passage from the URANS towards the LES region of flow detachment and at keeping the statistical region extent quite significant around the body.

\section{Test-case Description}

\subsection{Experimental set-up}

The experiments were conducted at the NASA Langley Basic Aerodynamic Research Tunnel (BART), which is a subsonic, atmospheric wind tunnel. The free stream velocity was set to $56 \mathrm{~m} / \mathrm{s}$ and in a second experiment to $44 \mathrm{~m} / \mathrm{s}$. The diameter of the cylinders in the second experiment was made higher to accommodate more pressure orifices, to be able to have a more precise pressure distribution around the cylinder. Therefore both velocities correspond to a Reynolds number based on the cylinder diameter of $\mathrm{Re}=166,000$. The free stream turbulence level at these conditions is about $0.09 \%$. Figure 1 shows schematically the configuration with the Tandem Cylinders placed in the wind tunnel, as well as a sketch of the computational used. 

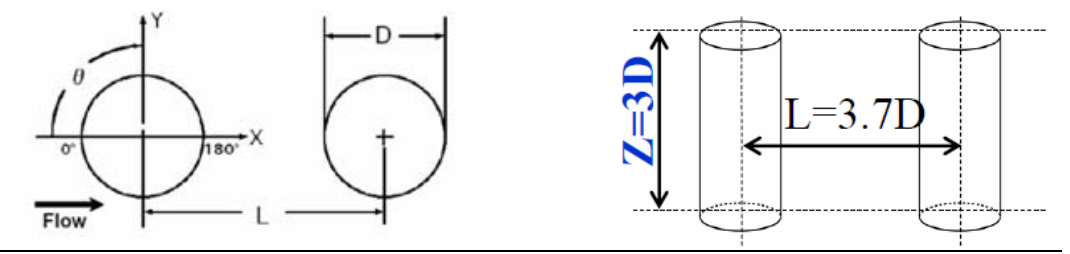

Fig. 1. Schematic representation of the tandem cylinders cross section and of the computational domain size

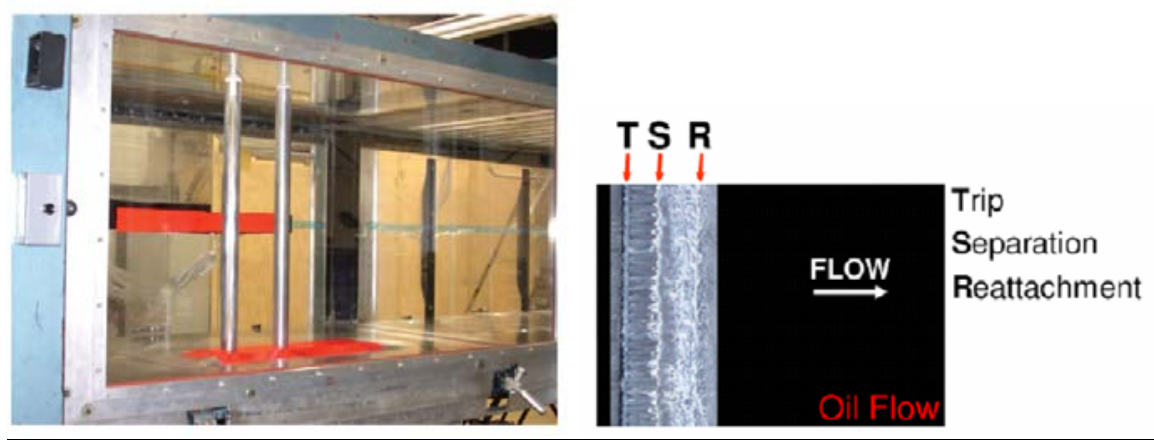

Fig. 2. Geometry of the test section, BARTH wind tunnel (Jenkins et al, 2005) and spanwise flow visualisation.

An oil flow visualisation is presented in fig. 2. Transition strips were taped on the cylinder between $\theta=50^{\circ}-60^{\circ}$ and $\theta=300-310^{\circ}$. Experiments were performed to demonstrate the effectiveness of these strips, where the cp-curve of a single cylinder with transition strips was compared to the cp-curve of a high Reynolds test-case at $\mathrm{Re}=8,4 \times 10^{6}$ of a single cylinder (Roshko, 1961). The distance between the cylinders is $\mathrm{L}=3: 7 \mathrm{D}$ (Figure 5.1 (a)) and the length of each cylinder is $16 \mathrm{D}$. The transition strips were placed on the first cylinder, as the second cylinders boundary is already fully turbulent from the stagnation point. This is because of the turbulence caused by the flow separation at the first cylinder. The Mach number of the experiment is $\mathrm{M}=0.128$, corresponding to an incompressible flow.

\subsection{Numerical method and turbulence modelling}

The simulations have been performed with the NSMB solver, (Navier-Stokes Multi Block), based on structured grids architecture. IMFT is part of the NSMB 
Fourth Symposium on Hybrid RANS-LES Methods, 28-30 September 2011, Beijing, China

consortium (Vos et al, 1998), contributing with turbulence modelling development for strongly detached unsteady aerodynamic flows. For the present simulations, central difference scheme has been applied, among the variety of spacial discretisation schemes available in the solver, after performing detailed tests (Barbut, 2010, Gual, 2011). Dual time stepping is used for the temporal discretisation.

The computational grid used is the one provided by NTS - St Petersbourg, by the partners M. Strelets and M. Shur in the ATAAC European program, shown in figure 3. The mesh refinement can be appreciated in Figure 3 (lower). The closest point to the wall at the second cylinder is at a distance of $\mathrm{y}=\mathrm{D}=3.0 \times 10^{-5}$.
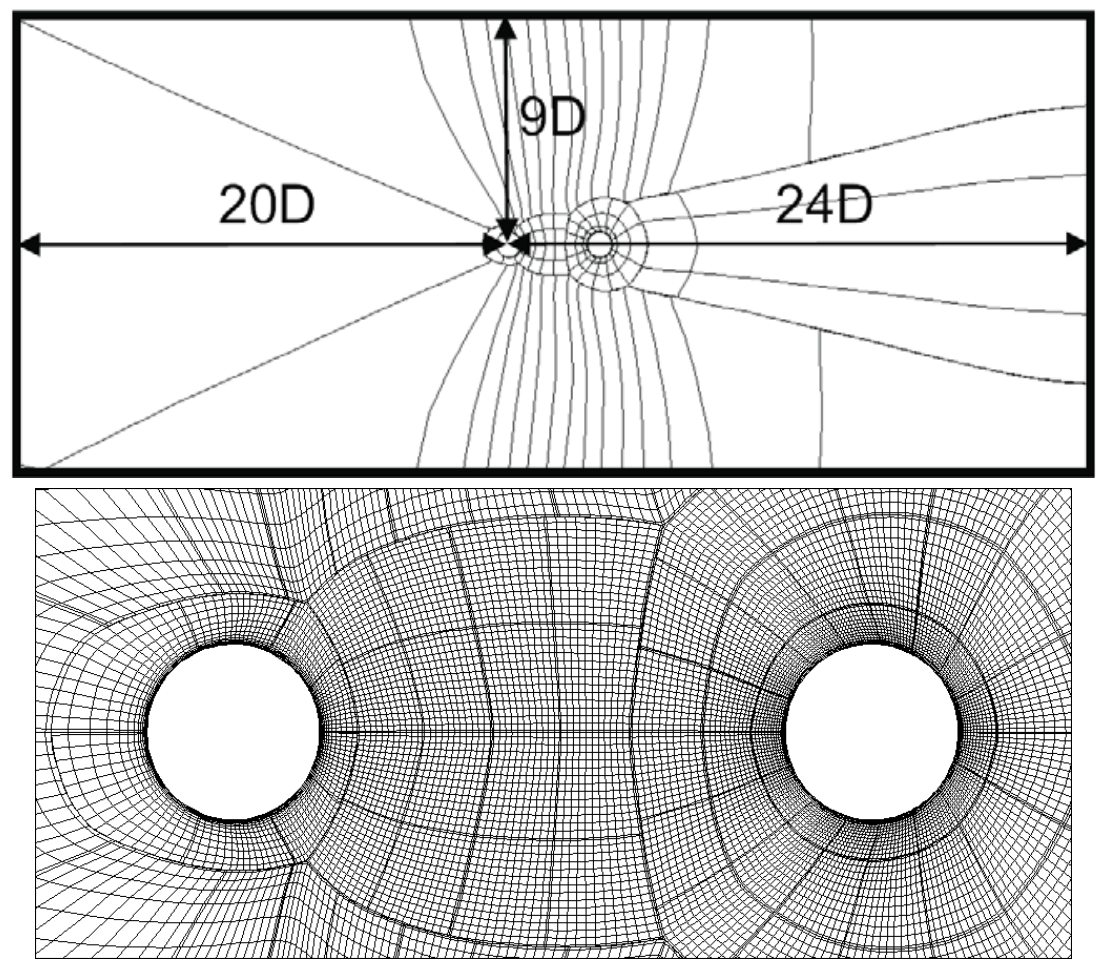

Fig. 3. Computational grid provided by NTS - St Petersbourg, Russia, for the ATAAC EU program partners

The present grid is of $16 \mathrm{M}$ points. The NSMB code is fully parallelised for MPI supercomputing architectures and benefits from parallelisation optimisation by means of the PRACE European initiative. The computations, using 512 to 1024 parallel processors in the present study, have been carried out at the SGI Altix supercomputer JADE of CINES, (Centre Interuniversitaire National de l'Enseignement Supérieur) and at the supercomputer CURIE of the CEA (Commisariat d'Etudes Atomiques) of France. 
The time step should be able to allow resolution of frequencies corresponding to a Strouhal number of order $\mathrm{St}=0.25$ and of higher frequencies predominant peaks due to Kelvin-Helmholtz eddies, responsible for the acoustic noise. A comparison with two time-step values has been performed in the present study, using dimensionless time steps $\Delta \mathrm{t}=0.01$ and 0.0005 . Recent results with an intermediate time step $\Delta \mathrm{t}=0.005$ are also provided. The computations with the coarser time step have been carried out over a long physical time corresponding to time samples 600 CTU (Cycle Time Units) and needed 100,000 CPU hours in the CINES 'JADE' Supercomputing centre, where the computation of the finer time step $(0.0005)$ has reached an order of six times lower duration at this stage.

The influence on the aerodynamic forces and on capturing the predominant frequencies is discussed.

The turbulence modelling is the DDES approach, in which the RANS turbulence length scale is improved by means of the Organised Eddy Simulation (Braza et al, 2006). It is recalled that distinction of coherent and chaotic turbulent structures is performed in OES by dual spectrum splitting, solving the ensemble-averaged Navier-Stokes equations, where the turbulence stresses are modelled by modified turbulence scales modelling. This has been be derived from DRSM (Differential Reynolds Stress Transport Modelling) and takes into account modification of the turbulence stresses in respect of non-equilibrium turbulence. The URANS turbulence length scale in the $k$ transport equation of DDES is chosen as the $l_{O E S}$ length scale, in the same way as reported in Bourguet et al. The turbulence viscosity has been chosen as the minimum between the eddy-viscosity provided by the $k$ - $\omega$-SST (Menter, 1994) and the $k$ - $\omega$-OES models.

\subsection{Results}

In figure 4, comparison of two DDES models is performed, using the more economic time step, $\Delta \mathrm{t}=0.01$. The results obtained show a rich turbulence statistical content, as well as formation of alternating shear layers and the wrap around mechanism towards the second cylinder. It can be seen that the DDESOES modelling provides finer shear layers, governed by the shedding of KelvinHelmholtz vortices, responsible for the acoustic noise (figure 4, right) and the contouring mechanism of the shear-layer vortices around the second cylinder, in accordance with the experiments. The 3D instantaneous visualisation of the flow field is shown in figures 5 and 6, where the complex vortex dynamics of finerscale chaotic structures interacting with the coherent structures is illustrated.
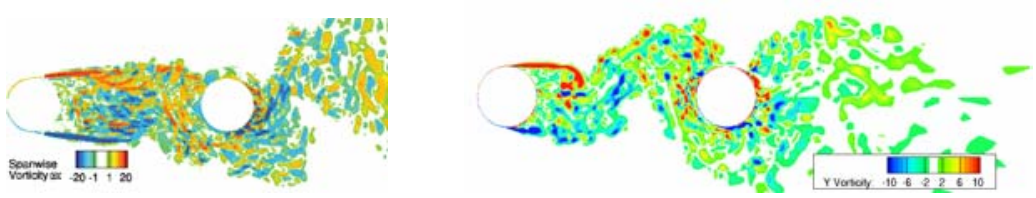

Fig. 4. Instantaneous spanwise vorticity cut (middle section), left: DDES- $k-\omega$-SST, right: DDES- $k-\omega$-OES 


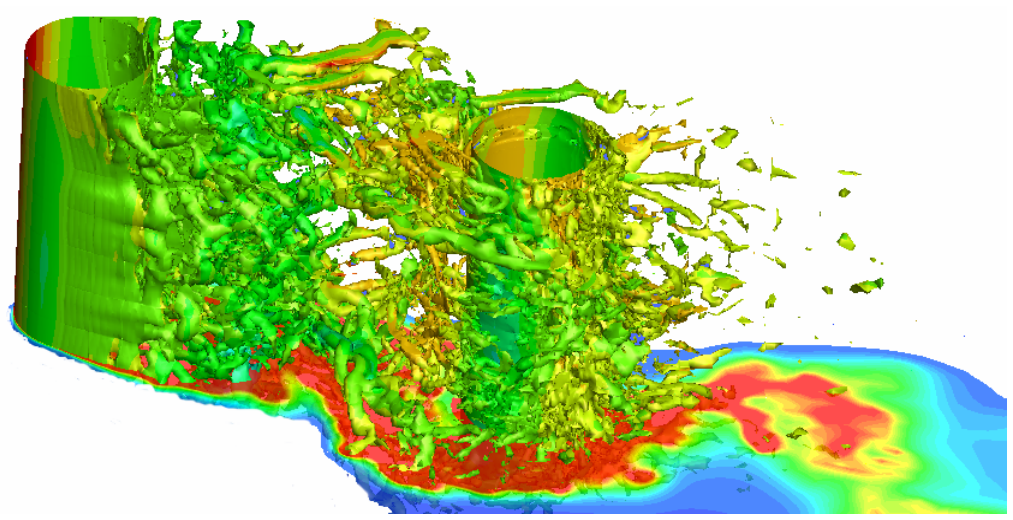

Fig. 5. Instantaneous 3D vorticity coloured by iso-pressure contours

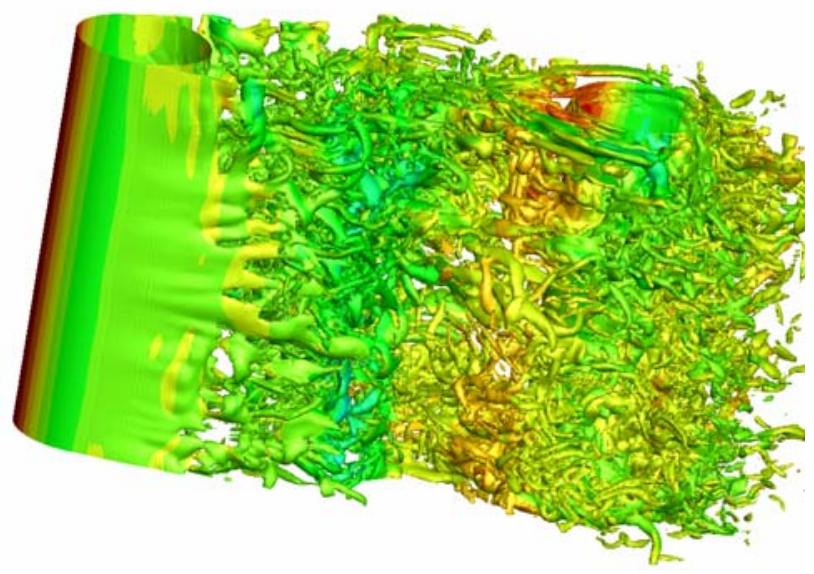

Fig. 6. Isovorticity $\Omega / D=15$ with pressure flood (red = high pressure, blue =low pressure)

Figure 7 (up) shows an instantaneous iso-velocity field, illustrating the rich statistical content obtained by the DDES-OES simulation and the surrounding turbulent fluid towards the second cylinder and in the downstream region, where the Kelvin-Helmholtz vortices are also visible. The averaged streamlines field is shown in figure 7 (down) in comparison with the experiment. A good agreement with the experiments is achieved. 

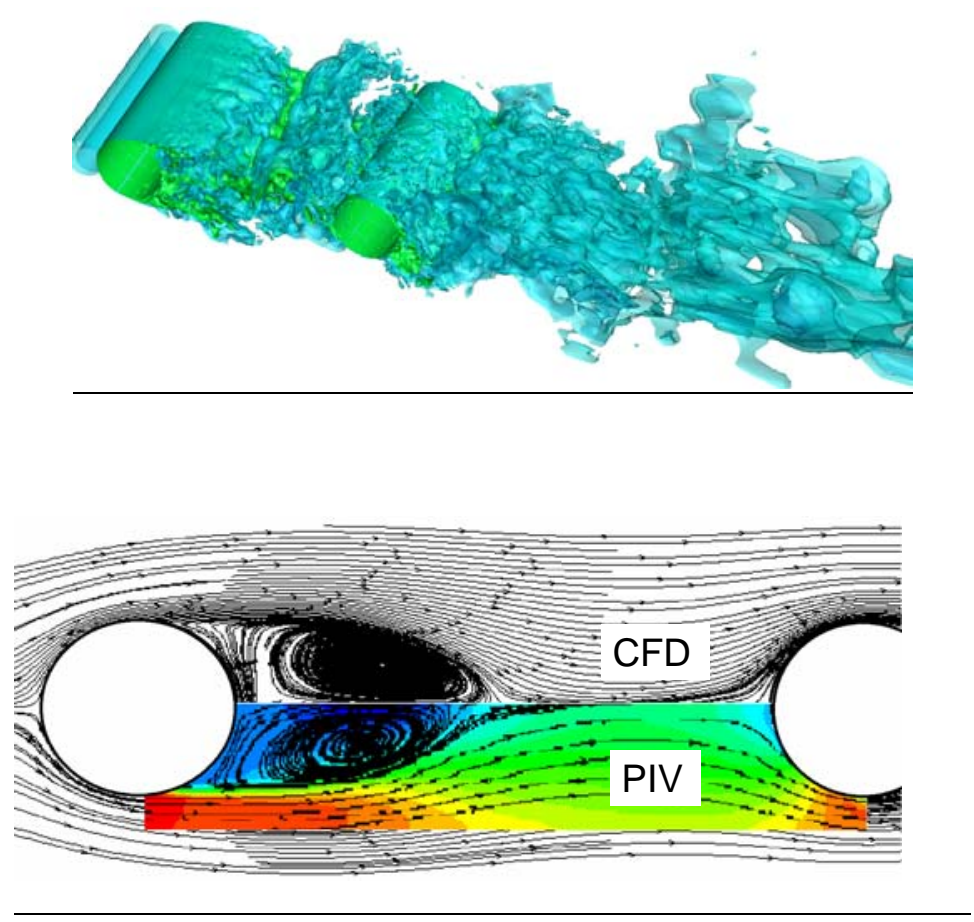

Fig. 7. View of the instantaneous velocity field (up) and of the averaged streamlines field (down) in comparison with the experiment of Jenkins et al.

Figure 8 shows the averaged velocity in the rear axis, sensitive to the flow detachment, where it is shown that a reasonably good agreement with the experiment is achieved. Figure 9 (up) shows the averaged $C p$ coefficient corresponding to the coarser time step, $\Delta t=0.01$ in comparison with the experiments. Concerning the first cylinder, the averaged maximum pressure values are lower than in the experiment, where a better agreement is obtained for the second cylinder. Figure 9 (down) shows the averaged pressure coefficient corresponding to the finest time step, $\Delta t=0.0005$, where the pressure peaks values are quite closer to the experimental ones. The computations need to be continued to achieve fully convergent statistics in the pressure plateau regions. 

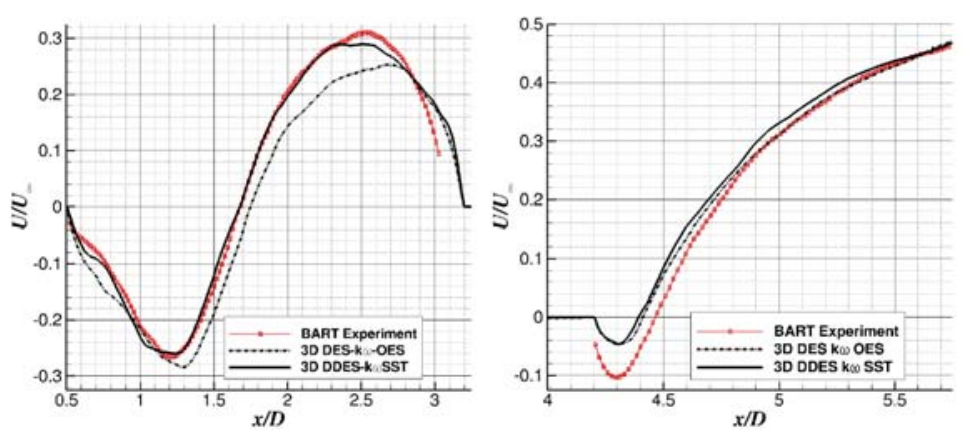

Fig. 8. Averaged velocity profiles in the gap region and at the rear flow
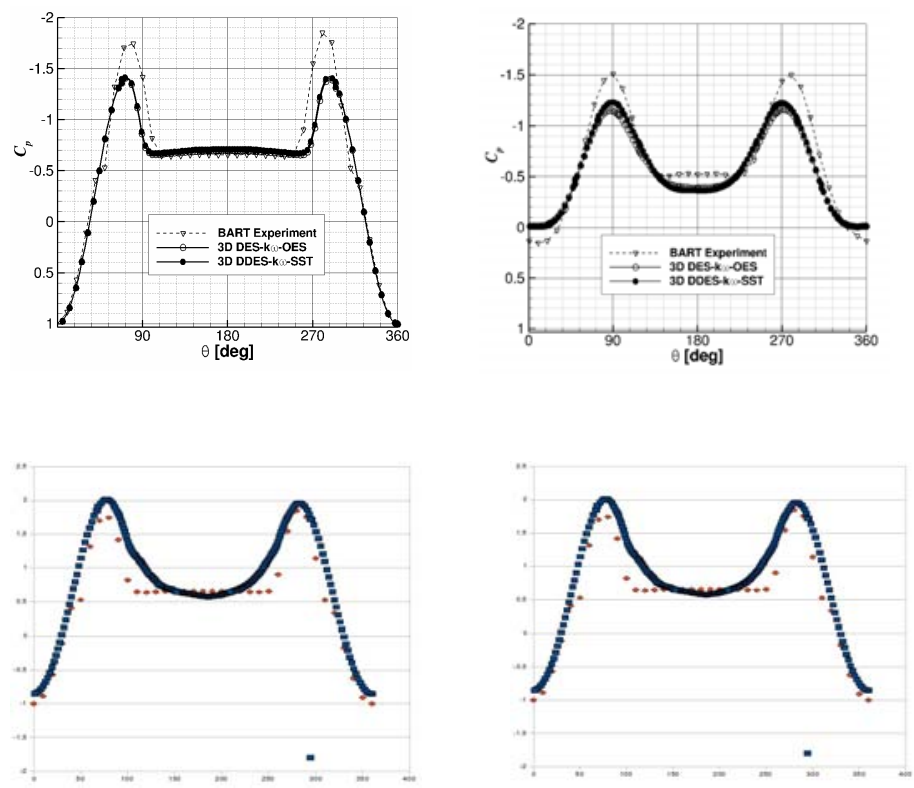

Fig. 9. Iall pressure coefficient $(C p)$ on the first (left) and second cylinder (right), compared with the experiment. Up: $\Delta t=0.01$. Down: $\Delta t=0.0005$.

The influence of the time step on the mean drag coefficient has been studied. The averaged drag coefficient $C_{D}$ is found 0.628 with $\Delta t=0.01$ and 0.500 for $\Delta t=0.005$. 
Comparing to numerical median value evaluated from a number of thirteen different numerical simulations reported by Lockard, $(C D=0.484)$, it is shown that the finer time step provides a much closer drag coefficient to this value. The experimental drag is not available in the present experiments.

The turbulence spectra show the principal instability modes, among which the von Kármán mode, as well as formation of a spectral 'bump' corresponding to the Kelvin-Helmholtz eddies. Of course, the chaotic turbulence effect provides the fact that these eddies are governed by a random vortex smearing around a predominant $\mathrm{K}-\mathrm{H}$ mode. The turbulence spectra presented in fig.10, corresponding to the coarser and the finer time steps, illustrate this interaction effect of the random turbulence on the predominant frequencies.
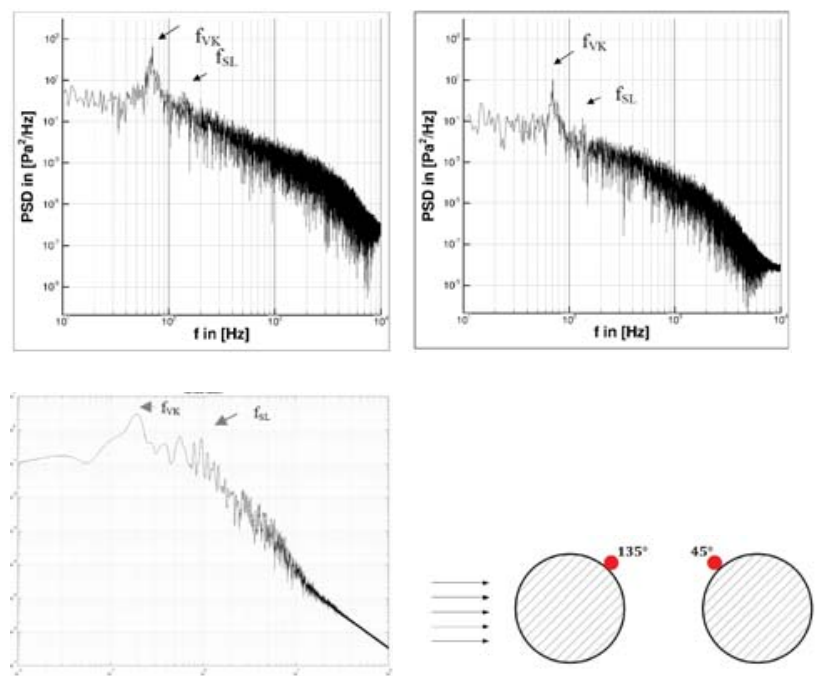

Fig. 7. Wall pressure spectra at the first and second cylinder, as in sketch, by means of $\Delta t=0.01$ (up) and $\Delta t=0.0005$ (down), first cylinder

Due to these facts and to the complex vortex flow system, the predominant frequencies are subjected to a modulation in time, that produces the aforementioned frequency bumps. As shown in the numerical flow visualizations (figures 5 to 7), the apparently chaotic flow structure is governed by a temporal coherence, interacting non-linearly with the random turbulence background.

A way to investigate the predominant frequencies modulation in time is the application of wavelet analysis to selected monitoring signals in the separated shear layers. The Morlet's wavelet analysis has been applied (for details of signal processing, see Marple, 1987, Grossman and Morlet, 1984). 

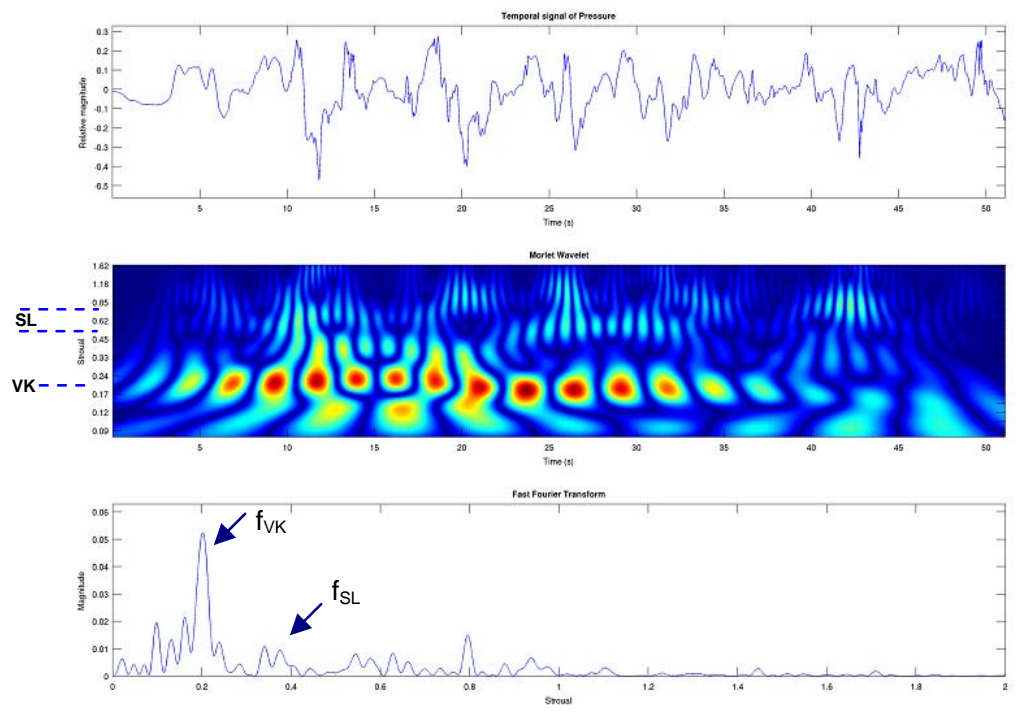

Fig. 8. Wavelet analysis on a pressure signal in the upper shear layer at $(x / D, y / D)=$ $(1.2,0.5)$. Up: signal versus time, middle, Morlet wavelets and dimensionless frequency versus time, down: dimensionless frequency versus time by means of FFT

The wavelet analysis shows the regions of maximum energy as a function of time. The vertical scale in the middle figure displays also the units in terms of dimensionless frequency. The lower array of cells in the Morlet wavelet (red kernels) indicate the modulation of the Strouhal number (Von Kármán frequency VK) versus time, where the upper array of cells indicates the width (frequency spreading) and modulation versus time of the shear layer mode (SL). In the time intervals $(10,15)$ and $(20,25)$, a merging of the lower path's energy towards the upper path is shown, corresponding to the non-linear interaction of these two modes.

\section{Conclusions}

A numerical study has been carried out to analyse the complex vortex pattern around two cylinders in tandem at Reynolds number 166,000, by means of Hybrid Turbulence modelling approaches. The Delayed Detached Eddy Simulation DDES-SST and DDES-OES models have been applied and comparison of results with the experiment is carried out. Furthermore, the influence of the 
computational time step has been studied on the same grid, provided by NTS - St Petersbourg. The results have shown that the flow structure between the two cylinders and past the second cylinder are well produced by the present methods. The statistical content of the DDES-OES is more detailed and the shear layer interfaces are finer and contain a number of Kelvin-Helmholtz vortices. Moreover, the spectral analysis shows that the flow is mainly governed by the von Kármán mode. A reasonably good agreement with the experiments carried out in the NASA-Langley Research Center is shown, especially concerning the mean streamlines structure, velocities and predominant frequencies. The influence of the time step on the flow parameters shows an improvement of the pressure coefficient and of the mean drag (compared with numerical results in the literature), with the finer time step. The computations with the finer time step are continued to provide statistics including more than a decade of periods of the main vortex shedding. The interaction between the Von Kármán mode and the shear layer frequencies bump is quantified by means of advanced signal processing, especially using wavelet analysis. The present study shows the ability of the DDES to simulate the present complex flow dynamics and predominant modes, associated with landing gear aerodynamic noise.

\section{Acknowledgement}

This work is part of the ATAAC research program, "Advanced Turbulence Simulations for Aerodynamic Application Challenges”, coordinated by DLRGermany (D. Schwamborn). The authors are grateful for the use of the French supercomputing facilities of CINES, IDRIS, CEA, this last in the context of the PRICE EU initiative. The authors thank F. Déliancourt, Master's degree student in IMFT and to Dr. T. Deloze, post-doc IMFT for their valuable contributions.

\section{References}

G. Barbut, PhD Thesis, «Analyse physique par simulation numérique d'écoulements turbulents instationnaires autour de surfaces portantes fixes ou en mouvement, à nombres de Reynolds et de Mach élevés », INPT, Institut National Polytechnique de Toulouse, 27 September 2010.

R. Bourguet, M. Braza, G. Harran, R. El Akoury (2008), J. Fluids and Structures, 24 (8), 1240-1251, 2008.

M. Braza, R. Perrin, Y. Hoarau (2006) “Turbulence Properties in the cylinder wake at high Reynolds number”, J. Fluids and Structures, 22, pp. 757-771

Grossmann, A., Morlet, J., Decomposition of Hardy functions into square integrable wavelets of constant shape, SIAM J., 1984.

M. Gual-Skopek, "Turbulence Modelling for Strongly Detached Turbulent Flows", Diploma Thesis Dissertation, IMFT-TUB, Toulouse, August 23rd 2011, supervisors M. Braza, F. Thiele, T. Höll. 
Fourth Symposium on Hybrid RANS-LES Methods, 28-30 September 2011, Beijing, China

M. Gual-Skopek, "Hybrid RANS-LES Modelling on a strongly detached turbulent flow", Aerodays, 30 March- ${ }^{\text {st }}$ April 2011, Madrid, Spain, $3^{\text {rd }}$ Undergraduate/Graduate Award by the Council of European Aerospace Societies (CEAS).

L. N. Jenkins, M. R. Khorrami, M. M. Choudhari and C. B. McGinley, AIAA 2005-2812, 2005.

M. R. Khorrami, D.P. Lockard, M. M. Choudhari, L. N. Jenkins, D. H. Neuhart, C. B. McGinley, "Simulations of bluff body flow interaction for noise source modelling”.AIAA Conference paper $\mathrm{N}^{\circ}$ 3203, Reno, 2006.

D.P. Lockard, M.R. Khorrami, M.M. Choudhari, F.V. Hutcheson, T.F. Brooks, D.J. Steed, "Tandem Cylinder Noise Predictions", 13th AIAA/CEAS Aeroacoustics Conference (28th AIAA Aeroacoustics Conference) AIAA 2007-3450.

D.P. Lockard, "Summary of the Tandem Cylinders solution from the benchmark problems for Airframe noise computations I - Workshop, 2007.

Marple S. L., Digital Spectral Analysis Prentice-Hall, Englewood Cliffs, 1987.

Menter, F. R., AIAA Journal, Vol. 32, No. 8, August 1994, pp. 1598-1605.

Vos J., Chaput E., Arlinger B., Rizzi A., and Corjon A., 1998 Recent advances in aerodynamics inside the NSMB (Navier-Stokes Multi-Block) consortium. In /36th Aerospace Sciences Meeting and Exhibit/, AIAA Paper 1998-0802, Reno, USA.

Vos, J., Leyland, P., V. van Kemenade, C. Gacherieu, N. Duquesne, P. Lotstedt, C. Weber, A. Ytterstrom, C. Saint Requier: NSMB Handbook 4.5 + Update for 6.0, Release 6.0, Code Handbook, 1998.

Wilcox, D.C. Turbulence Modelling for CFD, DCW Industries, California, USA, 1994 